\title{
Ventilación no invasiva en insuficiencia respiratoria aguda
}

\author{
JUAN SEPÚLVEDA S. ${ }^{1}$, JUAN ANDRÉS CARRASCO O. ${ }^{2}$, ANDRÉS CASTILLO M. ${ }^{2}$, \\ GUILIANA CÓRDOVA L. ${ }^{2}$, PATRICIO VALLE M. ${ }^{2}$, JOSÉ RODRÍGUEZ C. ${ }^{2}$ \\ 1. Residente de Pediatría. Pontificia Universidad Católica de Chile. \\ 2. Unidad de Paciente Crítico Pediátrica. Hospital Clínico Pontificia Universidad Católica de Chile.
}

\begin{abstract}
Non-invasive ventilation in acute respiratory failure

Background: Non-invasive ventilation (NIV) has become a therapeutic tool for acute respiratory failure in pediatric patients. Objective: To describe NIV experience at a Pediatric Intensive Care Unit, identifying factors associated to modality failure. Methods: Patients cohort who received NIV in acute respiratory failure during 2005-2007. Recolection of clinical and gasometrical variables previous and subsequent to connection to NIV. Descriptive statistic and logistic regression analysis were performed. Results: 51 events of NIV, where $55 \%$ cases were congenital cardiopathies. NIV main indication was respiratory distress post-extubation (31.4\%). 15 patients (29.4\%) required CMV. In univariate logistic regression for variables, an independent association was found between $\mathrm{FiO} 2$ pre-connection and $\mathrm{FiO} 2$ subsequent to NIV connection, with necessity of CMV. $9 \%$ presented complications and mortality was not related to ventilatory modality. Conclusions: NIV is useful in pediatric patients with acute respiratory insufficiency, with low risk of complications. The variables associated with failure are easy to obtain and interpretate.

(Key words: non-invasive ventilation,acute respiratory failure).

Rev Chil Pediatr 2008; 79 (6): 593-599
\end{abstract}

\section{RESUMEN}

Introducción: La ventilación no invasiva (VNI) se ha convertido en una herramienta terapéutica en insuficiencia respiratoria aguda en pediatría. Objetivo: Describir la experiencia con VNI en una unidad de paciente crítico pediátrico, e identificar factores asociados con fracaso de la modalidad. Pacientes y Método: Cohorte de pacientes que recibieron VNI en contexto de insuficiencia respiratoria aguda durante el período 2005-2007. Registro de variables clínicas y gasométricas previo y posterior a la conexión a VNI. Se realizó estadística descriptiva y análisis de regresión logística. Resultados: Analizamos 51 eventos de VNI, 55\% en postoperatorio de cardiopatías congénitas. La principal indicación de VNI fue difícultad respiratoria post extubación $(31,4 \%)$. Quince pacientes $(29,4 \%)$ fracasaron, requiriendo VMC. Al realizar regresión logística univariada se encontró asociación independiente entre la $\mathrm{FiO}_{2}$ pre conexión y $\mathrm{FiO}_{2}$

Trabajo recibido el 17 de marzo de 2008, aceptado para publicación el 20 de octubre de 2008.

Correspondencia a:

Juan Sepúlveda S.

E-mail: jrsepulv@gmail.com 
posterior a la conexión a VNI, con la necesidad de VMC. Nueve porciento presentaron complicaciones. No se observó mortalidad asociada a la modalidad ventilatoria. Conclusiones: La VNI es útil en pacientes pediátricos insuficiencia respiratoria aguda y tiene un bajo riesgo de complicaciones. Las variables asociadas con fracaso de VNI son de fácil obtención e interpretación.

(Palabras clave: ventilación no invasiva, insuficiencia respiratoria aguda).

Rev Chil Pediatr 2008; 79 (6): 593-599

\section{Introducción}

La ventilación no invasiva (VNI) es una forma de entregar soporte ventilatorio a través de la nariz y/o boca por medio de una interfase $^{1,2}$, mejora la ventilación y la oxigenación, y disminuye las complicaciones asociadas a la intubación traqueal y a la ventilación mecánica convencional (VMC) ${ }^{3}$. Ha surgido en las últimas décadas como una alternativa de asistencia ventilatoria en insuficiencia respiratoria agu$\mathrm{da}^{3,4}$. Si bien la mayoría de la experiencia se ha extrapolado de trabajos realizados en adultos, en los últimos años se ha reportado su utilidad en pacientes pediátricos con insuficiencia respiratoria aguda de diversas etiologías ${ }^{5-15}$. Se ha publicado su utilidad en el retiro precoz de la $\mathrm{VMC}$, en la insuficiencia respiratoria post extubación y, recientemente, han aparecido reportes de su uso en el postoperatorio de niños con cardiopatías congénitas ${ }^{3,16-18}$, sin embargo, no existe un consenso respecto de qué pacientes pueden obtener un mayor beneficio con este tipo de apoyo ventilatorio, ni qué factores pueden señalar precozmente una respuesta inadecuada y la necesidad de $\mathrm{VMC}^{19-21}$.

Los objetivos de este trabajo son describir la experiencia de esta Unidad de Paciente Crítico Pediátrica (UPC) en VNI e identificar variables que pudieran estar relacionadas con el fracaso de esta técnica.

\section{Pacientes y Método}

Se revisaron los registros de la Unidad de Paciente Crítico Pediátrico del Hospital Clínico de la Pontificia Universidad Católica de Chile y del Equipo de Terapia Respiratoria para identificar los pacientes que recibieron VNI durante el período comprendido entre enero de 2005 y septiembre de 2007.

Se confeccionó un formulario ad-hoc con los datos demográficos, antecedentes mórbidos, el diagnóstico de ingreso, la modalidad de VNI (presión positiva de vía aérea [CPAP] o presión positiva de vía aérea en dos niveles [BiPAP]), los parámetros, la interfase, la duración de la VNI, y la necesidad de VMC ante fracaso de la VNI. Se registró la frecuencia respiratoria, cardíaca, presión arterial y saturometría de oxígeno antes y después de la conexión a VNI. La fracción inspirada de oxígeno $\left(\mathrm{FiO}_{2}\right)$, se midió con oxímetro a través de conexión en $\mathrm{Y}$ al circuito; y los gases, arteriales o venosos, se midieron con equipo al lado de la cama del paciente (I-Stat) también fue registrada antes y después de la conexión a VNI.

Se consignaron los controles de signos vitales pre y post conexión que estuvieran en relación a una evaluación de gases arteriales o venosos.

También se registraron las complicaciones asociadas con la VNI y la duración de la estadía en la UPC.

Los antecedentes mórbidos relacionados con el uso de VNI fueron clasificados en 4 grupos: cirugía por cardiopatía congénita (incluye a los pacientes con cirugías paliativas o correctoras), respiratorio (incluye a pacientes con asma y daño pulmonar crónico), compromiso neurológico (pacientes con parálisis cerebral) e insuficiencia respiratoria en el paciente inmunocomprometido (en tratamiento oncológico o posterior a trasplante de precursores hematopoyéticos $\mathrm{u}$ órganos sólidos) $)^{20}$.

Las indicaciones de conexión a VNI se clasificaron según tipo de insuficiencia respiratoria en hipoxémica (requerimiento de oxígeno mayor a $40 \%$ para mantener saturación mayor 
a $90 \%)^{15}$ e hipercárbica $\left(\mathrm{PaCO}_{2}>45 \mathrm{mmHg}\right)^{8}$. También, por condición clínica en los siguientes grupos: enfermedad de parénquima pulmonar (EPP), que incluye neumonía, atelectasias, edema pulmonar, SDRA; obstrucción bronquial (OB), que incluye crisis asmáticas y bronquiolitis; retiro electivo de VMC (RVMC); dificultad respiratoria post extubación (DRPE), definida como pacientes que presentan dificultad respiratoria clínica, con aumento del trabajo respiratorio y frecuencia respiratoria asociado con hipoxemia y/o hipercarbia, sin indicación absoluta de reintubación, a quienes se les ofreció una prueba con VNI. Se excluyen de este grupo los pacientes con patología glótica, quienes no fueron conectados a VNI.

La indicación de intubación y conexión a VMC (fracaso VNI) definida por deterioro clínico con aumento de la dificultad respiratoria y/o alteraciones gasométricas (ya descritas), fue determinada por el médico residente.

Se realizó estadística descriptiva del grupo completo (cohorte no concurrente) y luego por tipo de VNI, y por grupo de indicación de VNI. Se realizó prueba t de Student para datos pareados paramétricos para comparar las variables clínicas y gasométricas pre y post conexión a VNI, y $\chi^{2}$ para evaluar variables dicotómicas respecto del fracaso de VNI. Para establecer si existen diferencias entre los grupos de indicación de VNI, se utilizó el método de Kruskal-Wallis.
Para definir la asociación con el fracaso de la VNI de variables dicotómicas y continuas, se llevó a cabo un análisis de regresión logística univariado y luego multivariado, empleando en el modelo variables obtenidas del análisis univariado, con significación clínica. Dada la proporción de pacientes con cardiopatías congénitas, se excluyeron para el análisis de factores pronósticos los que presentaban cardiopatías cianóticas por la dificultad para interpretar los gases arteriales en el contexto del grupo estudiado. Se consideró como significativo un valor $p<0,05$. Para los cálculos se utilizó el programa G-Stat.

\section{Resultados}

Durante el período estudiado se utilizó VNI en 44 pacientes con un total de 51 episodios. Cada evento de VNI se analizó como un caso independiente.

Los datos generales del grupo se presentan en la tabla 1. Al agruparlos por patología de base predisponente, $55 \%$ de pacientes cursaban postoperatorio de cardiopatías congénitas, $27 \%$ presentaban patología pulmonar, $10 \%$ inmunosupresión (trasplante renal, quimioterapia, post trasplante de precursores hematopoyéticos), $4 \%$ patología neurológica y un $4 \% \sin$ patología de base.

Catorce pacientes $(27,5 \%)$ eran portadores

Tabla 1. Características de pacientes por tipo de VNI

\begin{tabular}{|c|c|c|c|}
\hline & \multirow[t]{2}{*}{ Total } & \multicolumn{2}{|c|}{ Modalidad } \\
\hline & & CPAP & BiPAP \\
\hline n (\%) & $51(100)$ & $9(17,7)$ & $42(82,3)$ \\
\hline Edad (meses) & $24,6(0,03-210)$ & $10,9(0,03-92)$ & $24,6 \quad(0,3-210)$ \\
\hline Sexo (razón H/M) & $27 / 24$ & $4 / 5$ & $23 / 19$ \\
\hline Peso $(k g)$ & $12,8 \pm 8,8$ & $10,4 \pm 7,5$ & $13,3 \pm 9,0$ \\
\hline Z-score Peso/Edad & $-1,06 \pm 1,69$ & $-1.00 \pm 0,97$ & $-1,07 \pm 1,81$ \\
\hline Z-score Peso/Talla & $-0,24 \pm 1,67$ & $0,36 \pm 0,94$ & $-0,43 \pm 1,82$ \\
\hline $\operatorname{IPAP}\left(\mathrm{cmH}_{2} \mathrm{O}\right)$ & - & घ & $14(10-19)$ \\
\hline $\operatorname{EPAP}\left(\mathrm{cmH}_{2} \mathrm{O}\right)$ & घ & $8(4-10)$ & $7,5(4-10)$ \\
\hline Requerimiento VMC & $15(29,4 \%)$ & $4(44,4 \%)$ & $11(26,2 \%)$ \\
\hline Días VNI & $2(0-32)$ & $1(0-3)$ & $2(0-32)$ \\
\hline Días UPC & $10,5(2-79)$ & $9(8-19)$ & $14,5(2-79)$ \\
\hline
\end{tabular}

Datos se presentan como media (rango) o promedio \pm desviación estándar. IPAP: presión inspiratoria; EPAP: presión espiratoria. VNI: ventilación no invasiva; VMC: ventilación mecánica convencional. UPC: unidad paciente crítico. 
de síndrome de Down, 5 pacientes $(9,8 \%)$ con antecedente de prematurez, y $1(2 \%)$ era portador de enfermedad neuromuscular congénita.

Presentaron insuficiencia respiratoria hipoxémica el $70,6 \%$ del total de pacientes e hipercárbica el 29,4\%.

En cuanto al uso de VNI, en 16 casos $(31,4 \%)$ se utilizó en contexto de DRPE, y en $15(29,4 \%)$ por patología de parénquima pulmonar; dentro de este grupo el diagnóstico más frecuente fue el de neumonía en un 21,6\% (tabla 2). Cabe destacar que el $19,6 \%$ se conectó a VNI como salida electiva de VMC.

Se requirió conexión a $\mathrm{VMC}$ en 15 casos (29,4\%), de los cuales el 47\% tenía síndrome de Down $(\mathrm{p}<0,05)$, el $73 \%$ se encontraba en post operatorio de cardiopatía congénita $(\mathrm{p}=0,08)$, y el $20 \%$ tenía enfermedad respiratoria previa. Al analizarlos por grupo de indicación, existe un mayor porcentaje de fracaso en el grupo DRPE (56,3\%). El análisis entre grupos con el método de Kruskal-Wallis no resultó significativo.

Las interfases utilizadas fueron $60 \%$ máscara nasobucal, $17 \%$ cánula nasofaríngea (utilizada exclusivamente en el grupo CPAP), $6 \%$ máscara nasal y $4 \%$ máscara facial. No se describió la interfase utilizada en un 13\%.

Cuatro pacientes $(9 \%)$ presentaron complicaciones: neumotórax ( 2 pacientes en postope- ratorio de cardiopatías congénitas, con uso de drenajes torácicos: transposición de grandes arterias y en arteria coronaria anómala), conjuntivitis (1) y sólo un paciente con conjuntivitis y erosión cutánea en los puntos de apoyo de la interfase, este último relacionado a un mayor tiempo de VNI. No hubo relación con las presiones utilizadas en los pacientes que presentaron neumotórax, ambos requirieron conexión a VMC.

La mortalidad de esta serie fue de 9,2\% (3 pacientes en contexto de sepsis secundaria con falla orgánica múltiple y uno con daño pulmonar crónico con bronquiectasias e hipersecreción que falleció posterior al traslado a otro centro), sin tener relación con la VNI y ocurrió con posterioridad al uso de ésta.

Al evaluar las variables pre y post conexión se observó un aumento significativo en la $\mathrm{PaO}_{2}$ post conexión $(\mathrm{p}=0,01)$, en el $50 \%$ de los pacientes de esta serie, y una tendencia a disminuir la frecuencia cardíaca que no alcanzó significación estadística $(p=0,05)$. Al analizar por tipo de VNI, el grupo CPAP requirió una mayor $\mathrm{FiO}_{2}$ post conexión $(\mathrm{p}=0,01)$ (tabla 3$)$.

Con respecto al análisis de factores pronósticos, excluyendo a 7 pacientes con cardiopatías congénitas cianóticas, la regresión logística univariada demostró que sólo la $\mathrm{FiO}_{2}$ pre y post

Tabla 2. Características de pacientes por indicación de VNI

\begin{tabular}{|c|c|c|c|c|c|}
\hline & EPP & OB & RVMC & DRPE & FCR \\
\hline n (\%) & $15(29,4)$ & $9(17,6)$ & $10(19,6)$ & $16(31,3)$ & $1(2)$ \\
\hline Edad (meses) & $24,6(0,03-102)$ & $21,5 \quad(4,6-151)$ & $31,3 \quad(3,6-210)$ & $15,6(0,4-192)$ & 5,7 \\
\hline Sexo (razón H/M) & $7 / 8$ & $5 / 4$ & $5 / 5$ & $10 / 6$ & $0 / 1$ \\
\hline Peso $(\mathrm{kg})$ & $12,3 \pm 8,7$ & $12,5 \pm 8,0$ & $13,6 \pm 11,3$ & $13,3 \pm 8,3$ & 5,3 \\
\hline Z-score Peso/Edad & $-0,86 \pm 1,78$ & $-1,18 \pm 2,45$ & $-1,34 \pm 1,27$ & $-0,88 \pm 1,54$ & $-2,54$ \\
\hline Z-score Peso/Talla & $-0,34 \pm 2,22$ & $-1,22 \pm 0,94$ & $-0,77 \pm 1,65$ & $0,77 \pm 1,14$ & ND \\
\hline CPAP/BiPAP & $1 / 14$ & $1 / 8$ & $2 / 8$ & $4 / 12$ & $1 / 0$ \\
\hline IPAP $\left(\mathrm{cmH}_{2} \mathrm{O}\right)$ & $12(10-18)$ & $14(10-15)$ & $13(10-19)$ & $14(12-18)$ & NC \\
\hline $\operatorname{EPAP}\left(\mathrm{cmH}_{2} \mathrm{O}\right)$ & $6(4-8)$ & $8(6-8)$ & $7(4-10)$ & $8(4-10)$ & 4 \\
\hline Requerimiento VMC (\%) & $4(26,7)$ & $1(11,1)$ & $1(10)$ & $9(56,3)$ & 0 \\
\hline Días VNI & $2(0-11)$ & $2(0-9)$ & $5,5(1-32)$ & $1,5(0-4)$ & 1 \\
\hline Días UPC & $10(2-35)$ & $8(3-23)$ & $25,5(7-79)$ & $11(5-31)$ & ND \\
\hline
\end{tabular}

Datos se presentan como media (rango) o promedio \pm desviación estándar. EPP: enfermedad de parénquima pulmonar; OB: obstrucción bronquial; RVMC: retiro electivo de ventilación invasiva; DRPE: dificultad respiratoria post extubación; FCR: falla centro respiratorio. IPAP: presión inspiratoria; EPAP: presión espiratoria. VNI: ventilación no invasiva; VMC: ventilación mecánica convencional. UPC: unidad paciente crítico. ND: no determinado; NC: no corresponde. 
Tabla 3. Estadística descriptiva por tipo de VNI

\begin{tabular}{|c|c|c|c|c|c|c|c|c|c|}
\hline Variable & Pre-VNI & $\begin{array}{c}\text { Total } \\
\text { Post-VNI }\end{array}$ & $\mathbf{p}$ & Pre-VNI & $\begin{array}{c}\text { CPAP } \\
\text { Post-VNI }\end{array}$ & $\mathbf{p}$ & Pre-VNI & $\begin{array}{l}\text { BIPAP } \\
\text { Post-VNI }\end{array}$ & $\mathbf{p}$ \\
\hline Frecuencia respiratoria (cpm) & $43 \pm 19$ & $42 \pm 24$ & 0,78 & $51 \pm 18,3$ & $51 \pm 17,6$ & 0,9 & $41 \pm 19,2$ & $40=$ & 0,78 \\
\hline Frecuencia cardíaca (cpm) & $134 \pm 28$ & $125 \pm 27$ & 0,05 & $146 \pm 20,9$ & $146 \pm 12,2$ & 0,9 & $131 \pm 29$ & $121 \pm 27,5$ & 0,05 \\
\hline Presión arterial sistólica $(\mathrm{mmHg})$ & $100 \pm 19$ & $102 \pm 18$ & 0,43 & $89 \pm 21,7$ & $88 \pm 20,1$ & 0,53 & $102 \pm 17,8$ & $105 \pm 16,2$ & 0,28 \\
\hline Presión arterial diastólica $(\mathrm{mmHg})$ & $56 \pm 14$ & $57 \pm 12$ & 0,21 & $49 \pm 12,6$ & $47 \pm 10,1$ & 0,64 & $57 \pm 13,7$ & $59 \pm 11,8$ & 0,08 \\
\hline Presión arterial media $(\mathrm{mmHg})$ & $71 \pm 15$ & $73 \pm 18$ & 0,25 & $64 \pm 14,9$ & $62 \pm 18$ & 0,78 & & 75 & 0,16 \\
\hline Saturación de $\mathrm{O}_{2}(\%)^{a}$ & $87 \pm 12$ & $87 \pm 12$ & 0,64 & $84 \pm 18,3$ & $87 \pm 11$ & 0,4 & $87 \pm 10,5$ & $87 \pm 12$ & 0,93 \\
\hline $\mathrm{FiO}_{2}(\%)^{\mathrm{a}}$ & $54 \pm 23$ & $56 \pm 20$ & 0,42 & $48 \pm 13,3$ & $58 \pm 13$ & 0,01 * & $56 \pm 24$ & $56 \pm 21$ & 0,85 \\
\hline $\mathrm{PaO}_{2}(\mathrm{mmHg})^{\mathrm{a}}$ & $57 \pm 22$ & $68 \pm 44$ & 0,01 * & $52 \pm 29$ & $66 \pm 32$ & 0,24 & $58 \pm 20$ & $68 \pm 46$ & 0,32 \\
\hline $\mathrm{PaCO}_{2}(\mathrm{mmHg})$ & $45 \pm 11$ & $44 \pm 12$ & 0,52 & $46 \pm 8,4$ & $44 \pm 7,1$ & 0,53 & $45 \pm 12$ & $44 \pm 12,4$ & 0,74 \\
\hline Bicarbonato $(\mathrm{mmol} / \mathrm{L})$ & $27 \pm 5,7$ & $28 \pm 6,5$ & 0,68 & $26 \pm 2,1$ & $26 \pm 2,4$ & 0,76 & $27 \pm 6,2$ & $27 \pm 7,0$ & 0,74 \\
\hline Exceso de base & $1,3 \pm 5,5$ & $1,4 \pm 6,2$ & 0,58 & $0,7 \pm 2,2$ & $0,4 \pm 1,9$ & 0,64 & $1,5 \pm 6$ & $1,6 \pm 6,8$ & 0,51 \\
\hline
\end{tabular}

Datos se presentan como promedio \pm desviación estándar. ${ }^{a}$ Excluidos los pacientes con cardiopatías cianóticas.

Tabla 4. Regresión logística univariada

\begin{tabular}{|c|c|c|c|c|}
\hline & Variable & $\begin{array}{l}\text { Cambio de riesgo } \\
\text { (\%/unidad de variable) }\end{array}$ & $\mathbf{p}$ & R2 Nagelkerke \\
\hline \multirow{11}{*}{ 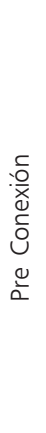 } & Frecuencia respiratoria & & 0,83 & \\
\hline & Frecuencia cardíaca & & 0,60 & \\
\hline & Presión arterial sistólica & & 0,97 & \\
\hline & Presión arterial diastólica & & 0,55 & \\
\hline & Presión arterial media & & 0,95 & \\
\hline & Saturación de $\mathrm{O}_{2}$ & & 0,76 & \\
\hline & $\mathrm{FiO}_{2}$ & $+4,86 \%$ & 0,01 & 0,28 \\
\hline & $\mathrm{PaO}_{2}$ & & 0,23 & \\
\hline & $\mathrm{PaCO}_{2}$ & & 0,84 & \\
\hline & Bicarbonato & & 0,26 & \\
\hline & Exceso de base & & 0,21 & \\
\hline \multirow{11}{*}{ 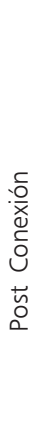 } & Frecuencia respiratoria & & 0,06 & \multirow{11}{*}{0,20} \\
\hline & Frecuencia cardíaca & \multirow{10}{*}{$+4,68$} & 0,83 & \\
\hline & Presión arterial sistólica & & 0,45 & \\
\hline & Presión arterial diastólica & & 0,88 & \\
\hline & Presión arterial media & & 0,82 & \\
\hline & Saturación de $\mathrm{O}_{2}$ & & 0,08 & \\
\hline & $\mathrm{FiO}_{2}$ & & 0,02 & \\
\hline & $\mathrm{PaO}_{2}$ & & 0,44 & \\
\hline & $\mathrm{PaCO}_{2}$ & & 0,35 & \\
\hline & Bicarbonato & & 0,70 & \\
\hline & Exceso de base & & 0,37 & \\
\hline
\end{tabular}

conexión se asociaron significativamente con el fracaso de VNI ( $p<0,01$ para ambas variables). La edad no se asoció con mayor riesgo de fracaso $(p=0,89)$. Al reunir estas variables en un modelo multivariado, no se encontró asociación significativa (tabla 4).

\section{Discusión}

Este trabajo presenta una serie de pacientes diferente a otras publicadas, heterogénea en cuanto al tipo de pacientes incluidos, de un amplio rango etario, y con un suficiente número 
de casos. Al tratarse de un centro de referencia de resolución de cardiopatías congénitas, destaca el grupo de pacientes cardiópatas post operados, quienes presentan escenarios variables de interacciones cardiopulmonares y en quienes un soporte ventilatorio menos controlado pudiera eventualmente ser considerado menos seguro, con algunas escasas referencias en la literatura ${ }^{17,18}$.

La indicación de VNI es comparable a la literatura, salvo por una mayor proporción de uso en dificultad respiratoria post extubación en lo que no están incluidos los pacientes con compromiso glótico como se señaló en la descripción de la metodología.

El aumento en la $\mathrm{PaO}_{2}$ post conexión es concordante con el efecto deseado de la VNI, $\mathrm{y}$, al igual que la tendencia a disminuir la frecuencia cardíaca reflejarían el mejor estado de oxigenación de los pacientes con el soporte no invasivo. En nuestra opinión, ésta es la mejor demostración de la utilidad de la VNI.

La frecuencia de fracaso en esta serie está dentro de lo reportado en la literatura, y si bien no se encontraron diferencias estadísticamente significativas en relación al grupo de indicación, destaca la mayor frecuencia de fracaso en el grupo de dificultad respiratoria post extubación y en el grupo de síndrome de Down que presenta mayores dificultades desde el punto de vista de vía aérea y de requerimientos de sedación $^{20,21}$. La edad de los pacientes no presentó una asociación estadística con un mayor riesgo de fracaso en los pacientes menores, lo que pudiera explicarse por los criterios de selección de VMC de la unidad.

Los fracasos de la VNI se presentaron en forma precoz, dentro de las primeras horas luego de la conexión y no se relacionaron con la mortalidad de la serie. Los 4 pacientes que fallecieron lo hicieron muy posterior al uso de VNI, no teniendo sus causas de muerte ninguna relación causal con ella.

Las variables asociadas a fracaso de VNI $\left(\mathrm{FiO}_{2}\right.$ antes y después de conexión a VNI) definidas por el análisis de regresión logística son parámetros clínicos utilizados a diario en las unidades críticas. El comportamiento de estas variables es concordante y presenta lógica clí- nica. En el modelo multivariado no se lograron determinar variables asociadas, lo que probablemente pueda estar influido por en número de pacientes y de eventos de fracaso de VNI. En este punto, la indicación de VNI puede ser importante, así como el tipo de VNI utilizado. Otros grupos han encontrado asociación con una $\mathrm{FiO}_{2}>80 \%$ a la hora de iniciada la $\mathrm{VNI}^{21}$ y con el diagnóstico de distrés respiratorio agudo y mayor puntaje PELOD ${ }^{20}$, elementos no reportados por esta serie.

Cabe destacar la baja frecuencia de complicaciones cutáneas y conjuntivales de este grupo a diferencia de otras series ${ }^{19-21}$. Esto se explica por el cuidadoso manejo de enfermería en el adecuado sello de la interfase, que puede ser particularmente difícil en pacientes más pequeños. Ambos neumotórax reportados ocurrieron en el contexto de pacientes con cirugía torácica y uso de drenajes, no estando relacionadas con las presiones administradas a la vía aérea.

\section{Conclusión}

La VNI es útil en el paciente crítico, presentando en nuestra serie un bajo porcentaje de complicaciones. Requiriere una evaluación constante y precoz de la evolución para determinar su continuación o cambio a una modalidad invasiva. No existe aún un consenso respecto de qué pacientes deben recibir VNI. Existe una amplia gama de patologías relacionadas con fracaso del método siendo en nuestra serie la DRPE la más importante, descartando de ellas el compromiso glótico. En nuestro grupo una mayor $\mathrm{FiO}_{2}$ previa y posterior a la conexión a VNI se asoció al fracaso del método. Se requieren estudios prospectivos de mayor tamaño para definir el rol de otras variables.

\section{Agradecimientos}

Al Dr. Jaime Cerda L., por su valiosa colaboración en el análisis estadístico y la interpretación de los resultados. 


\section{Referencias}

1.- Cheifetz I: Invasive and Noninvasive Pediatric Mechanical Ventilation. Respir Care 2003; 48: 442-53.

2.- Pons Ódena M, Cambra Lasaosa F: Ventilación mecánica (III). Weaning, complicaciones y otros tipos de ventilación. Ventilación no invasiva. An Pediatr (Barc) 2003; 59: 165-72.

3.- Evans T, International consensus conferences in intensive Care medicine: Non-invasive positive pressure ventilation in acute respiratory failure. Am Respir Crit Care Me. 2001; 163: 283-91.

4.- Liesching T, Kwok H, Hill N: Acute applications of noninvasive positive pressure ventilation. Chest. 2003; 124: 699-713.

5.- Fortenberry JD, Del Toro J, Jefferson L, Evey L, Haase $D$ : Management of pediatric acute hypoxemic respiratory insufficiency with bilevel positive pressure (BiPAP) nasal mask ventilation. Chest 1995; 108: 1059-64.

6.- Padman $R$, Lawless $S$, Kettrick $R$ : Noninvasive ventilation via bilevel positive airway pressure support in pediatric practice. Crit Care Med 1998; 26: 169-73.

7.- Nørregaard $O$ : Noninvasive ventilation in children. Eur Respir J 2002; 1332-42.

8.- Teague $W G$ : Noninvasive ventilation in the pediatric intensive care unit for children with acute respiratory failure. Pediatr Pulmonol 2003; 35: 418-26.

9.- Akingbola O, Hopkins R: Pediatric noninvasive positive pressure ventilation. Pediatr Crit Care Med 2001; 2: 164-69.

10.- Bourguignon D, Krepel F, Troster E: Noninvasive ventilation in pediatrics. J Pediatr (Rio J) 2003; 79 (Suppl 2): S161-68.

11.- Niranjan $V$, Bach $J$ : Nonivasive management of pediatric neuromuscular ventilatory failure. Crit Care Med 1998; 26: 1061-65.

12.- Thill P, McGuire J, Baden H, Green T, Checchia P: Noninvasive positive-pressure ventilation in children with lower airway obstruction. Pediatr Crit Care Med 2004; 5: 337-42.

13.- Akingbola O, Simakajornboon N, Hadley E, Hopkins $R$ : Noninvasive pressure-ventilation in pediatric status asthmaticus. Pediatr Crit Care Med 2002; 3: 1814.

14.- Piastra M, Antonelli M, Caresta E, Chiaretti A, Polidori $G$, Conti $G$ : Noninvasive ventilation in childhood acute neuromuscular respiratory failure: a pilot study. Respiration 2006, 73: 791-8.

15.- Prado F, Godoy MA, Godoy M, Boza ML: Ventilación no invasiva como tratamiento de la insuficiencia respiratoria aguda en Pediatría. Rev Méd Chile 2005; 133: $525-33$.

16.- Rodríguez JA, Von Dessauer B, Duffau G: Utilización de la CPAP de forma no invasiva en la laringitis postextubación del paciente pediátrico. Estudio controlado y aleatorizado. Arch Bronconeumol 2002; 38: 463-7.

17.- Tokuda Y, Matsumoto M, Sugita T, Nishizawa J: Nasal mask bilevel positive airway pressure ventilationfor diaphragmatic paralisis after pediatric open-heart surgery. Pediatr Cardiol 2004; 25: 5523.

18.- Chin K, Takahashi K, Ohmori K, et al: Noninvasive ventilation for pediatric patients under 1 year of age after cardiac surgery. J Thorac Cardiovasc Surg 2007; 134: 260-1.

19.- Medina A, Prieto S, Los Arcos M, et al: Aplicación de ventilación no invasiva en una unidad de cuidados intensivos pediátricos. An Pediatr (Barc) 2005; 62: 1319.

20.- Essouri S, Chevret L, Durand P, Haas V, Fauroux B, Devictor D: Noninvasive positive pressure ventilation: Five years of experience in a pediatric intensive care unit. Pediatr Crit Care Med 2006; 7: 329-34.

21.- Bernet V, Hug M, Frey B: Predictive factors for the success of noninvasive mask ventilation in infants and children with acute respiratory failure. Pediatr Crit Care Med 2005; 6: 660-4. 